\title{
Material dependence of ${ }^{2} \mathrm{H}(\mathrm{d}, \mathrm{p})^{3} \mathrm{H}$ cross section at the very low energies
}

\author{
Ali İhsan Kılıçc ${ }^{1}$ Konrad Czerski ${ }^{2,3}$, Fadime Kuştan-Kılıç ${ }^{2,4}$, Natalia Targosz-Sleczka ${ }^{2}$, Daniel Weissbach ${ }^{2,3}$, Armin Huke ${ }^{3}$, \\ and Götz Ruprecht ${ }^{2}$
}

\author{
${ }^{1}$ University of Guelph, 50 Stone Road E, Guelph, Canada \\ ${ }^{2}$ University of Szczecin Department of Physics, Szczecin, Poland \\ ${ }^{3}$ Institute for Nuclear Solid-State Physics, Berlin, Germany \\ ${ }^{4}$ University of Gazi, Department of Physics. 06500 Teknikokullar, Ankara, Turkey
}

\begin{abstract}
Calculations of the material dependence of ${ }^{2} \mathrm{H}(\mathrm{d}, \mathrm{p})^{3} \mathrm{H}$ cross section and neutron-to-proton branching ratio of $\mathrm{d}+\mathrm{d}$ reactions have been performed including a concept of the $0^{+}$threshold single particle resonance. The resonance has been assumed to explain the enhanced electron screening effect observed in the $\mathrm{d}+\mathrm{d}$ reaction for different metallic targets. Here, we have included interference effects between the flat and resonance part of the cross section, which allowed us to enlighten observed suppression of the neutron channel in some metals such as $\mathrm{Sr}$ and $\mathrm{Li}$. Since the position of the resonance depends on the screening energy that strongly depends on the local electron density. The resonance width, observed for the $d+d$ reactions in the very hygroscopic metals ( $\mathrm{Sr}$ and $\mathrm{Li}$ ) and therefore probably contaminated by oxides, should be much larger than for other metals. Thus, the interference term of the cross section depending on the total resonance width provides the material dependences.
\end{abstract}

\section{Introduction}

The ${ }^{2} \mathrm{H}(\mathrm{d}, \mathrm{p}){ }^{3} \mathrm{H}$ and ${ }^{2} \mathrm{H}(\mathrm{d}, \mathrm{n}){ }^{3} \mathrm{He}$ reactions have been investigated from beginnings of physics [1]. The $d+d$ reactions are especially important for developing the next generation of power plants [2], understanding of the nuclear process within stars [3], exploring the physics of few-body nucleon systems [4], solving some astrophysics problems [5] and the problems of nucleon nucleoninteraction [6].

The exponential-like increase of the reaction crosssection observed for decreasing projectile energies, as compared to the cross section for bare nuclei, can be described corresponding to the reduction of the Coulomb barrier height by a screening energy. The theoretical values of the large screening energies are by at least a factor of two smaller than experimentally determined [7]. The enhanced electron screening effect recently observed in the $\mathrm{d}+\mathrm{d}$ fusion and some other nuclear reactions in the metallic environments is essential for general understanding of stellar reaction rates white and brown dwarfs or giant planets since it can strongly increase the nuclear reaction rates in dense astrophysical plasmas [8]. The angular distributions and the branching ratio between the ${ }^{2} \mathrm{H}(\mathrm{d}, \mathrm{p})^{3} \mathrm{H}$ and ${ }^{2} \mathrm{H}(\mathrm{d}, \mathrm{n})^{3} \mathrm{He}$ reactions strongly depend on the target material, being different self-implanted deuterized metallic environments. Whereas for metallic targets made of Al, Zr, Ta both observable show the same projectile energy dependence as in the case of the gas target measurements, for $\mathrm{Sr}$ and $\mathrm{Li}$ a significant suppression of the neutron channel and increase of its angular anisotropy could be observed for deuteron energies below $20 \mathrm{keV}$ [9].

To describe these effects, we applied an alternative model based on the T-matrix elements [10] which corresponds to broad ${ }^{4} \mathrm{He}$ resonance contributions. Within this approach, interference effects between resonances could be easily included. The theoretical results agree very well with experimental data obtained for usual materials and width multichannel R-matrix theory [11]. To explain the experimental data obtained for unusual materials, it was necessary to include a new $0^{+}$threshold resonance. This resonance should have a single particle deuteron-deuteron structure and be very narrow (the total width of few eV). The contribution of the $0^{+}$resonance to the reaction cross section could reduce large screening energies observed. 


\section{Single particle $0^{+}$threshold resonance}

A hypothetical $0^{+}$resonance in ${ }^{4} \mathrm{He}$ placed very close to the $d+d$ reaction threshold has been proposed [12]. The high energy tail of the resonance would contribute to the observed enhancement of the cross-section due to the screening effect and reduce experimental screening energy down to the theoretical value the mathematical point of view a coherent sum of two reaction amplitudes reads as follows:

$$
\left|A_{1} e^{i \emptyset_{1}}+A_{2} e^{i \emptyset_{2}}\right|^{2}=A_{1}^{2}+A_{2}^{2}+2 A_{1} A_{2} \cos \left(\emptyset_{1}-\emptyset_{2}\right)(1)
$$

Where $\emptyset_{1}$ and $\emptyset_{2}$ are nuclear phase shifts. The above procedure can be applied to the coherent sum of cross sections resulting from the narrow threshold $0^{+}$ resonance $\left(\sigma_{R}\right)$ and from broad overlapping resonances giving a flat cross section $\left(\sigma_{F}\right)$ contribution.

Interferences in the total cross section are possible only between resonances of the same spin and parity. Thus, the interference part of the total cross section relates only to $0^{+}$resonances, both a narrow postulated one and the broad resonances of the flat contribution. Therefore, since cross section in this consist of two components, it can be written as

$\sigma\left(0^{+}\right)=\sigma_{F}\left(0^{+}\right)+\sigma_{R}+2 \sqrt{\sigma_{F}\left(0^{+}\right)} \sqrt{\sigma_{R}} \cos \left(\emptyset_{1}-\emptyset_{2}\right)$

$\sigma_{F}\left(0^{+}\right)$corresponds to $\alpha_{0}$ transition matrix elements [10]. Since the Deuteron energy of about $10 \mathrm{keV}$ is much larger than the resonance energy and the resonance width, expression for the resonance phase shift can be simplified

$$
\tan \emptyset_{R}=\frac{\Gamma}{2\left(E-E_{R}\right.}, E \gg E_{R} \quad E \gg \Gamma \quad \emptyset_{R}=\frac{\Gamma}{2 E} \ll 1
$$

therefore

$\cos \left(\emptyset_{F}-\emptyset_{R}\right)=\sin \emptyset_{F} \sin \emptyset_{R}+\cos _{\mathrm{F}} \cos _{\mathrm{R}} \approx \frac{\Gamma}{2 E} \sin \emptyset_{F}+$ $\sqrt{1-\frac{\Gamma^{2}}{4 E^{2}}} \cos \emptyset_{F}$

expanding the square root value to the first term in Taylor series we get:

$\cos \left(\emptyset_{F}-\emptyset_{R}\right) \approx \frac{\Gamma}{2 E} \sin \emptyset_{F}+\left(1-\frac{1}{2} \frac{\Gamma^{2}}{4 E^{2}}\right) \cos \emptyset_{F} \approx$

$\frac{\Gamma}{2 E} \sin \emptyset_{F}+\cos \emptyset_{F}$

the final expression was obtained by neglecting the quadratic term. Since the $\frac{\Gamma}{2 E}=10^{-4}$ for $\mathrm{E}=10 \mathrm{keV}$ we can simplify the expression further

$\cos \left(\emptyset_{F}-\emptyset_{R}\right) \approx+\cos \emptyset_{F}$ total cross section is given by

$$
\sigma_{T o t}=\frac{1}{\sqrt{E}} \frac{1}{\sqrt{E_{G}}} P(E) S(E)
$$

where $P$ and $S$ denote the s-wave penetration factor and the astrophysical $S$-factor, respectively. The total $S$-factor can be expressed by a sum of $S_{a}$ and $S_{b}$ arising from the isotropic and an isotropic contribution. Roughly, $\frac{1}{3}$ of the $L=0$ partial cross section (corresponding to $S_{a}$ ) comes from the $0^{+}$matrix element $\sigma_{0}$. Therefore, the $0^{+}$flat cross section contribution is given by

$$
\sigma_{F}\left(0^{+}\right)=\frac{1}{3} \sqrt{\frac{1}{E_{c m} E_{G}}} \mathrm{P}(\mathrm{E}) \mathrm{S}_{\mathrm{a}}
$$

here, $E_{G}$ represents Gamow energy, equal for $986 \mathrm{keV}$ for the $\mathrm{d}+\mathrm{d}$ system. $E$ is the energy of the deuteron in the center of mass system, and can be expressed by the wave number $\mathrm{k}$, we get:

$\sigma_{F}\left(0^{+}\right)=\frac{1}{3} \frac{\pi}{k} \mathrm{P}(\mathrm{E}) \frac{\sqrt{2 m}}{\hbar} \frac{1}{E_{G}} S_{a}$

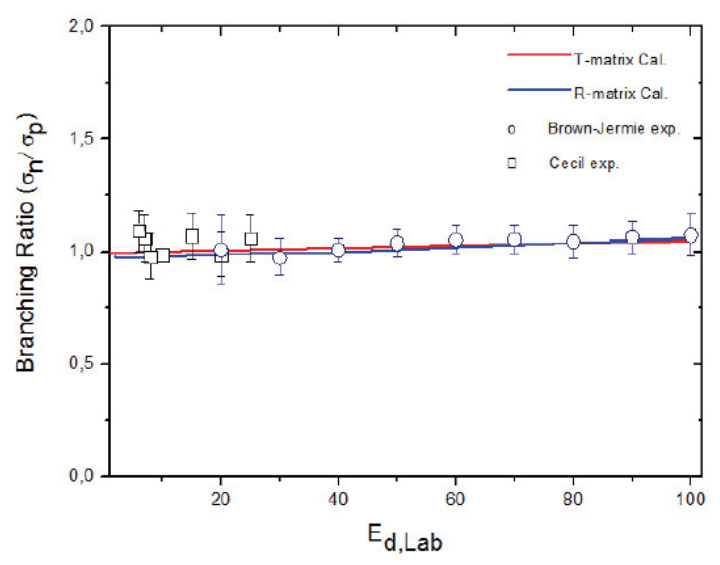

Fig. 1. Experimental neutron-to-proton branching ratio obtained for targets of $\mathrm{Ta}, \mathrm{Sr}$, and $\mathrm{Li}$ [9] compared to the calculations assuming deuteron polarization (blue-line), and without polarization(red line)

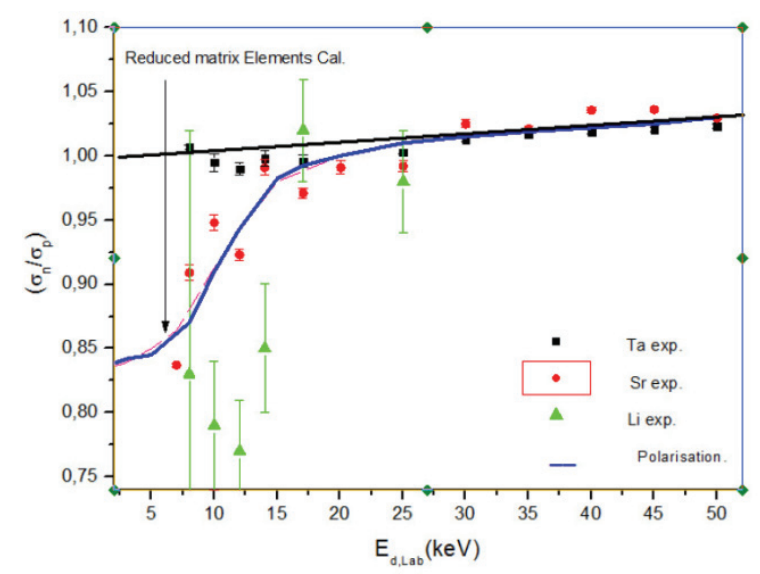

Fig. 2. Experimental and theoretical neutron-to-proton branching ratio. The experimental data are from [16] and [17]. 
For the resonance contribution, we apply Breight-Wigner formula of the proton channel (similar for the neutron channel)

$\sigma_{R}=\frac{\pi}{k^{2}} \frac{\Gamma_{d} \Gamma_{p}}{\left(E-E_{0}\right)^{2}+\frac{\Gamma^{2}}{4}} \approx \frac{\pi}{k^{2}} \frac{\Gamma_{d} \Gamma_{p}}{E^{2}}$

The $\mathrm{d}+\mathrm{d}$ reaction cross section can be enhanced by a hypothetical $0^{+}$resonance state in $\mathrm{He}^{4}$ compound nucleus. Due to its molecular structure [13], it can be described as a single-particle resonance width:

$$
\Gamma_{d}(E)=2 k_{d} a P\left(E_{d}\right) \frac{\hbar^{2}}{\mu a^{2}}|\Theta|
$$

The $\mathrm{d}+\mathrm{d}$ reaction cross section can be enhanced by a hypothetical $\mathrm{O}^{+}$resonance state in $\mathrm{He}^{4}$ compound nucleus. Due to its molecular structure [13], it can be described as a single-particle resonance width:

Here, $a=7 \mathrm{fm}$ is the reaction channel radius. $\mu$ is reduced mass of deuteron, $|\Theta|^{2}$ stays for the dimensionless reduced width being equal to unity for the single- particle resonance. Taking account that $\mathrm{E} \gg E_{R}$, $\Gamma \gg \Gamma_{d}$ and we put the $\Gamma_{d}$ to $\sigma_{R}$.

Finally, the resonance cross section reads as follows:

$\sigma\left(0^{+}\right)=\frac{\pi}{k} P(E)\left[A^{2} \frac{1}{3} S_{a}+\frac{B \Gamma_{p}}{E^{2}}+2 A \sqrt{\frac{S_{a}}{3}} \sqrt{\frac{B \Gamma_{P}}{E}} \cos \emptyset_{F}\right.$

Where

$A^{2}=\frac{2}{3 \pi} \frac{\sqrt{m}}{\hbar} \frac{1}{E_{G}}, \quad \mathrm{~B}=2 \frac{2 \hbar^{2}}{\mu a}$

Due to the destructive interference effects, we can now reduce $\sigma_{0}$ matrix elements [10] in the low energy region as it is necessary to explain the observed material dependence of the branching ratio Fig. 1. The material dependence can be additionally enhanced assuming a smaller neutron partial width of the resonance. The branching ratio can be calculated using formula 12. for neutron channel and proton channel separately. In usual case, $\Gamma_{n}, \Gamma_{p}$ are much smaller than for $\mathrm{Sr}$ and Ta. Therefore, branching ratio can be described without resonance contribution (Fig. 2).

\section{Conclusions}

In the present work, we could show, how the material dependence of the branching ratio of the $\mathrm{d}+\mathrm{d}$ fusion reaction might be explained applying the concept of the threshold resonance. The branching ratio does not depend on the penetration factor any more. The screening effect should not directly influence its value. In the usual case, $\Gamma_{n}$ and $\Gamma_{p}$ also do not depend on the target material as they are only proportional to the nuclear wave functions. The destructive interference could be also observed in the energy dependence of the enhancement factor obtained in the new UHV experiment on the $\mathrm{Zr}$ target [14]. The resonance contribution reduces the estimated screening energy by a constant factor, making it closer to the theoretical value. The direct resonance evidence can be expected by the precision measurements of the $d+d$ reactions at deuteron energies below $5 \mathrm{keV}$ where different energy dependence of the enhancement factor due to electron screening and the resonance could much more visible. Corresponding experiments are planned at the University of Szczecin applying the new accelerator facility with a UHV target chamber [15].

\section{References}

1. E. Lawrence et al., Phys. Rev. Lett.44, 56 (1933).

2. R.M. More, Phys. Rev. Lett 51396 (1983)

3. E. Margaret et al, Rev. Mod. Phys. 29547 (1957)

4. $\quad$ S.E. Koonin and M. Mukerjee, Phys. Rev. C42 (1990) 1639.

5. D. Kirkman et al., Apj S, 1491.6 (2003) 1639.

6. Angula et al., Z. Phys. A345 (1993) 231.

7. A. Huke et al. Phys. Rev. C 78 (2008) 015803

8. Zs. Faulaop et al., Nucl. Phys. A 758 (2005) 697

9. A. Huke et al. Eur. Phys. J A 27 (2006) 187-192

10. H. Schieck. Eur. Phys. J A 44 (2010) 321354

11. H.S. Bosch, G.M. Hale. Nucl. Fusion 32 (1992) 1919

12. N.Targozh-Sleczka et al. Acta Phys. Pol. V42 (2011) 795-798

13. W.Von Oertzen Z. Phys. A 37 A44 (1996) 354

14. K. Czerski et al. J. Phys.G, Nucl. Part. Phys 35 (2008) 014012

15. M. Kaczmarski et al. Acta Phys.Pol. B 45 (2014) 509.

16. R.E. Brown and N. Jarmie Phys. Rev. C 41. (1990) 1391.

17. F.E. Cecil et al. Phys.Rev C 47 (1987) 1178 